\title{
Attributes of Infinity
}

\author{
Kiamran Radjabli* \\ Utilicast, La Jolla, California, USA. \\ * Corresponding author. Email: kradjabli@utilicast.com \\ Manuscript submitted May 15, 2016; accepted October 14, 2016. \\ doi: 10.17706/ijapm.2017.7.1.42-48
}

\begin{abstract}
The concept of infinity is analyzed with an objective to establish different infinity levels. It is proposed to distinguish layers of infinity using the diverging functions and series, which transform finite numbers to infinite domain. Hyper-operations of iterated exponentiation establish major orders of infinity. It is proposed to characterize the infinity by three attributes: order, class, and analytic value. In the first order of infinity, the infinity class is assessed based on the "analytic convergence" of the Riemann zeta function. Arithmetic operations in infinity are introduced and the results of the operations are associated with the infinity attributes.
\end{abstract}

Key words: Infinity, class, order, surreal numbers, divergence, zeta function, hyperpower function, tetration, pentation.

\section{Introduction}

Traditionally, the abstract concept of infinity has been used to generically designate any extremely large result that cannot be measured or determined. However, modern mathematics attempts to introduce new concepts to address the properties of infinite numbers and operations with infinities. The system of hyperreal numbers [1], [2] is one of the approaches to define infinite and infinitesimal quantities. The hyperreals (a.k.a. nonstandard reals) $* \mathbf{R}$, are an extension of the real numbers $\mathbf{R}$ that contains numbers greater than anything of the form $1+1+\ldots+1$, which is infinite number, and its reciprocal is infinitesimal. Also, the set theory expands the concept of infinity with introduction of various orders of infinity using ordinal numbers. The first transfinite ordinal, denoted $\omega$, is the order type of the set of nonnegative integers [3]. This is the "smallest" of Cantor's transfinite numbers, defined to be the smallest ordinal number greater than the ordinal number of the whole numbers, and is denoted with the notation $\omega=\{0,1, \ldots \mid\}$. The surreal number system [4], [5] represents an arithmetic continuum containing the real numbers as well as infinite and infinitesimal numbers, respectively larger or smaller in absolute value than any positive real number. The hyperreal numbers can be realized as subfields of the surreals. The surreal numbers contain all transfinite ordinal numbers and are constructed through forms inductively as equivalence classes of pairs of sets of surreal numbers, restricted by the condition that each element of the first set is smaller than each element of the second set. The arithmetical operations of surreal number forms, e.g. $\mathrm{X}=\left\{\mathrm{X}_{\mathrm{L}} \mid \mathrm{X}_{\mathrm{R}}\right\}$ and $\mathrm{y}=\left\{\mathrm{Y}_{\mathrm{L}}\right.$ $\left.\mid Y_{R}\right\}$, are defined by recursive formulas. The surreal numbers establish different orders of infinity using powers of transfinite ordinal, i.e. $\omega, \omega^{\omega}, \omega^{\omega^{\omega}} \ldots$

This paper presents a different approach to mathematical perception of infinity and its classification based on hyper-operations and Riemann zeta-function. The zeta function, $\zeta(\mathbf{s})$ is a function of a complex variable s, which analytically continues the sum of the infinite series and converges when the real part of $s>$ 
1. The Riemann zeta function is an important special function, which arises in definite integration. The zeta function has a unique analytic continuation to the entire complex plane, excluding the point $s=1$, which corresponds to a simple pole with complex residue [6]. For even arguments, the zeta function can be computed analytically.

\section{Transform to Infinity}

It is not possible to define the infinite number in the finite domain of numbers. This does not mean that infinite number does not exist, but only the fact that it cannot be defined within the known domain of finite numbers. Let's assume that infinite numbers do exist and can be defined in a different domain, but remain undefined and not "reachable" from the domain of finite numbers. Diverging function or series can be perceived as "infinity transform", which map the finite domain to the infinite domain (Fig. 1). The mathematics of finite numbers considers divergence as an approach to single infinite number, regardless of the type of the divergence. However, if infinite numbers exist in the infinite domain, then "infinity transform" may approach different infinite numbers. Thus, infinity numbers are perceived as ever changing dynamic values in infinite domain as opposed to the static values of finite numbers domain.

The infinite number cannot be "visualized" from the finite numbers perspective, but its divergence "trajectory" can be used for segregation of infinity into different layers. It is not possible to determine the precise infinite numbers using the expressions of finite domain. However, it may be possible to segregate the infinite numbers by assigning a category to the path yielding to infinite numbers. The extension of the single infinity number concept to "poly-infinity" also allows introduction of operations on infinite numbers. The actual resulting value of the operations on infinite numbers is undetermined, but the associated attributes can be established.

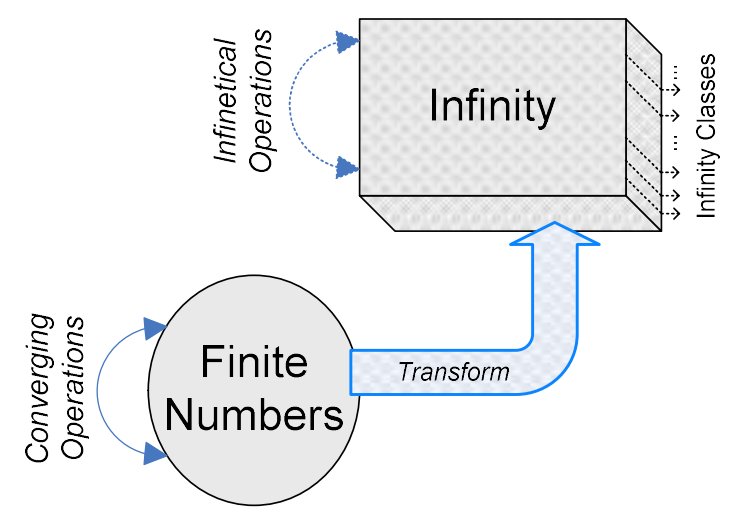

Fig. 1. Transform from finite to infinite domains.

\section{Infinity Classification}

The common definition of infinity is "a number greater than any assignable quantity or countable number." However, an unassignable quantity can still be compared to other unassignable quantities at any given moment of diverging process based on the significantly different quality of greatness. Two power functions of different power do not intersect anywhere in infinity for $\mathrm{x} \rightarrow \infty$, and therefore can be considered approaching infinity in different layers of infinity or effectively belong to different levels of infinity with respect to the intensity of their divergence.

It is proposed in [6] to distinguish infinity and infinitesimal based on the Riemann zeta-function [7] for an integer argument $\mathbf{s}$. 


$$
\zeta(s)=\sum_{n=1}^{\infty} \frac{1}{n^{s}}
$$

For $s=1$, the zeta function diverges, i.e. $\zeta(1)=\infty$. However, the following function resolves the asymptotic divergence of zeta function near the pole of zeta function [6].

$$
\Omega_{\zeta}(s)=\zeta(s)+\zeta(2-s)
$$

For $s=1, \Omega_{\zeta}(1)=\lim _{\Delta s \rightarrow 0} \zeta(1+\Delta s)+\zeta(1-\Delta s)=2 \gamma$

where $\boldsymbol{\gamma}$ - is Euler-Mascheroni constant

The following function allows to assign a value that can indirectly serve as a measurement of divergence intensity:

$$
Z(s)=\frac{2 \gamma-\zeta(s)-\zeta(2-s)}{2 \gamma-1} \operatorname{sign}(1-s)
$$

Let's associate (denoted as symbol below) the infinity of the power function $\mathrm{f}=\mathrm{ax}^{\mathrm{S}}$ with its integral, and subsequently, with the corresponding summation of the zeta function $\zeta(\mathbf{s})$ and its "analytic value" $\mathbf{Z}(\mathbf{s})$.

$$
\lim _{x \rightarrow \infty} a x^{s} \Rightarrow \sum_{n=1}^{\infty} \frac{1}{n^{-s}} \Rightarrow Z(-s)
$$

In spite of the fact that the infinity of the function does not have any directly assignable quantity or countable number, the analytical association with the zeta function provides a virtual finite value that can be assigned to the quality level of the divergence. For a power function, the infinity level $\mathbf{s}$ is considered to be "analytically converging" to the value of $\mathbf{Z ( s )}$.

Evidently, the power functions do not cover all transforms to infinity, which are mapped and associated with Riemann's zeta function $\zeta(\mathbf{s})$. Hyper-operations deliver much more intense divergences. The hyper-operation sequence is an infinite sequence of arithmetic operations that starts with the unary operation of successor (order 0 ), then continues with the binary operations of addition (order 1), multiplication (order 2), and exponentiation (order 3). After that, the sequence proceeds with further binary operations extending beyond exponentiation, using right-associativity. Hyper-operation [8] of forth order (hyper-4) is defined as s-times iterated exponentiation function, also known as "tetration" [9], which is presented in one of the following notations [3] as $\mathbf{s}$-th tetration of $\mathbf{x}$ :

$$
h_{4}(x, s)=x^{x^{x \cdots}}=x \uparrow \uparrow s=x \uparrow^{2} s=\left({ }^{s} x\right)
$$

For $s=2$, the tetration function $h_{4}(x, 2)=x^{x}$. The sequence of hyper-powers $x,{ }^{2} x,{ }^{3} x,{ }^{4} x \ldots=\left\{{ }^{S} x\right\}$ converges for all $\mathbf{x}$ in the interval $\left[\mathrm{e}^{-\mathrm{e}}, \mathrm{e}^{1 / \mathrm{e}}\right]$, and diverges for all $\mathbf{x}$ outside of that interval [8]. For $\mathrm{s} \rightarrow \infty$, the infinitely iterated tetration function $\mathrm{H}_{4}(\mathrm{x})=\lim _{s \rightarrow \infty} h_{4}(x, s)$ represents an extremely fast divergence to infinity when $\mathrm{x} \rightarrow \infty$, and subsequently $\mathrm{H}_{4}(\mathrm{x})$ can be related to the an ultra-high order of infinity.

Hyper-operations [8] of higher order can be also defined, e.g. "pentation" (hyper-5), which is iterated "tetration", and uses notation $\mathbf{x} \uparrow \uparrow \uparrow \mathbf{s}$ or $\mathbf{x} \uparrow \mathbf{3 s}$. The definition of similar hyper-operations can be extended to hyper-M, and ultimately to hyper- $\infty$, with infinite number of arrows in the notation, which is the infinite 
order of infinity.

Let's introduce the following function $\mathbf{K}(\mathbf{s})$ based on the tetration function $\mathrm{h}_{4}(\mathrm{n})$ for integers:

$$
K(s)=\sum_{n=1}^{\infty}\left({ }^{s} n\right)
$$

The function $\mathbf{K}(\mathbf{s})$ is similar to Riemann's zeta function $\zeta(\mathbf{s})$, and can be used to define ultra-high infinite numbers, where $s$ is positive integer. For $s=1, K(1)=\zeta(-1)=-\frac{1}{12}$, and for any integer $s>1$, it can be shown that $\mathbf{K}(\mathbf{s})$ is greater than corresponding Riemann's zeta function sums, i.e.

$$
\sum_{n=1}^{\infty}\left({ }^{s} n\right)>\sum_{n=1}^{\infty} \frac{1}{n^{-s}}
$$

Evidently, the zeta function cannot provide adequate categories of infinity for the hyper-operations and the relevant divergence to extremely high levels of infinity. For these ultra-high infinities, it is more appropriate to establish higher orders using the order of hyper-operations. Hyper-operations allow definition of infinite orders of infinity.

Most common mathematical operations used in the physical world belong to the first order of non-iterated operations at simple exponentiation of 3, i.e. $\mathbf{x} \uparrow \mathbf{s}$. In the first order, the zeta-function can be effectively used as a classification to distinguish infinity levels approached by the power functions, and the $\mathbf{Z}(\mathbf{s})$ function can be used to assign an analytic value to the infinity.

For hyper-operations, it may be also possible to find a function, which can serve as analytic value. For example, for tetration, the following function provides calculation similar to the function (2).

$$
\Omega_{H}(s, x)=\left({ }^{s} x\right)+\left({ }^{-s} x\right)
$$

A linear approximation of $\mathbf{s}_{\mathbf{X}}$ to the differentiability requirement is given by:

$$
\left({ }^{s} x\right)=\left\{\begin{array}{lr}
\log _{x}\left({ }^{s+1} x\right) & s \leq-1 \\
1+s & -1<s<0 \\
\left.x^{(s-1} x\right) & s>0
\end{array}\right.
$$

Then, for $s>1$

$$
\left.\Omega_{H}(s, x)=x^{(s-1} x\right)+\log _{x}\left({ }^{s+1} x\right)
$$

The Table 1 provides a list of infinity transforms and the corresponding infinity attributes to characterize uniquely the associated infinity numbers produced by the divergence. The analytic values are provided only for power functions, as analytic continuations of other diverging functions are yet to be clearly established.

\section{Operations in First Order of Infinity}

Since infinity is a domain of a different nature, it is conceivable that arithmetic of finite numbers may be not applicable in infinity and even mixing of finite and infinite numbers in the infinite domain may not be feasible as it is possible in the finite domain. To distinguish the arithmetic of undefined numbers in the infinite domain from the arithmetic of finite number, the term "infinetic" is used further below. Let us try to 
define basic manipulations with infinite numbers, i.e. summation, subtraction, multiplication, division. The result of the infinetic operation is still an undefined number, which belongs to infinite domain, but it has associated attributes: infinity order, class, and analytic value.

Table 1. Infinity Attributes for Diverging Transforms

\begin{tabular}{|l|l|l|l|}
\hline \multirow{2}{*}{ Transform } & \multicolumn{2}{|l|}{ Attributes } & \multicolumn{2}{l|}{ Class } & Analytic Value \\
\cline { 2 - 4 } & Order & 1 & $\begin{array}{l}\zeta(-1)=-\frac{1}{12} ; \\
Z(-1) \approx 0.23122\end{array}$ \\
\hline $\lim _{x \rightarrow \infty} x$ & 1 & 2 & $\begin{array}{l}\zeta(-2)=0 ; \\
Z(-2) \approx 0.46692\end{array}$ \\
\hline $\lim _{x \rightarrow \infty} x^{2}$ & 1 & $\mathrm{M}$ & $\begin{array}{l}\zeta(-\mathrm{M}) ; \\
\lim _{x \rightarrow \infty} x^{M}\end{array}$ \\
\hline $\lim _{x \rightarrow \infty} x^{x}$ & 1 & 1 & \\
\hline $\lim _{x \rightarrow \infty} x^{x^{x}}$ & 2 & 2 & \\
\hline $\lim _{x \rightarrow \infty}\left({ }^{N} x\right)$ & 2 & $\mathrm{~N}$ & \\
\hline $\lim _{x \rightarrow \infty}\left(x \uparrow^{3} x\right)$ & 3 & 1 & \\
\hline $\lim _{x \rightarrow \infty}\left(x \uparrow^{4} x\right)$ & 4 & 1 & \\
\hline
\end{tabular}

Let's look at the summation and subtraction through the "infinite transform" perspective. The power function transform to infinity is measured exclusively on the basis of the diverging intensity of $\mathbf{x}^{\mathbf{s}}$, because the finite coefficient $\mathbf{a}$, no matter how large, belongs to the finite domain, and therefore, coefficient a will be always less than the $x \rightarrow \infty$. Two power functions transform to infinite domain as follows:

$$
\begin{aligned}
& \lim _{x \rightarrow \infty} \sum_{i=1}^{N_{1}} a_{i} x^{s_{1}} \Rightarrow \operatorname{In} f\left(s_{1}\right)=Z\left(-s_{1}\right) \\
& \lim _{x \rightarrow \infty} \sum_{i=1}^{N_{2}} b_{i} x^{s_{2}} \Rightarrow \operatorname{Inf}\left(s_{2}\right)=Z\left(-s_{1}\right)
\end{aligned}
$$

where $s_{1}>s_{2}$.

Then, the summation and subtraction can be associated to Riemann's zeta function $\zeta(\mathbf{s})$ and the analytic value of $\mathbf{Z}(\mathbf{s})$ function through class comparison:

$$
\begin{aligned}
& \operatorname{Inf}\left(s_{1}\right) \oplus \operatorname{Inf}\left(s_{2}\right) \ominus Z\left(-s_{3}\right) \\
& \operatorname{Inf}\left(s_{1}\right) \ominus \operatorname{Inf}\left(s_{2}\right) \ominus Z\left(-s_{3}\right)
\end{aligned}
$$

where $s_{3}$ is the solution of the following equation for summation

$$
\lim _{x \rightarrow \infty}\left(x^{s}-\sum_{i=1}^{N_{1}} a_{i} x^{s_{1}}-\sum_{i=1}^{N_{2}} b_{i} x^{s_{2}}\right)=0
$$


where $\left(s_{1}+s_{2}\right)<s_{3}<1+\left(s_{1}+s_{2}\right)$

The encircled arithmetical symbols designate "infinetical" operations, i.e. arithmetical operations on infinite numbers.

Essentially, the proposed summation/subtraction of infinite operands result in the assignment of the new order, which is incremented/decremented by $s<1$ to the result of summation/subtraction.

The change of order $s \geq 1$ occurs in case of multiplication or division of infinite numbers. The multiplication and division through the "infinite transform" perspective can be also associated to Riemann's zeta function $\boldsymbol{\zeta ( \mathbf { s } )}$ and the analytic value of $\mathbf{Z}(\mathbf{s})$ function as follows:

$$
\begin{aligned}
& \operatorname{Inf}\left(s_{1}\right) \otimes \operatorname{Inf}\left(s_{2}\right) \ominus Z\left(-s_{1}-s_{2}\right) \\
& \operatorname{Inf}\left(s_{1}\right) \oslash \operatorname{Inf}\left(s_{2}\right) \ominus Z\left(-s_{1}+s_{2}\right)
\end{aligned}
$$

The proposed approach of association with Riemann's zeta function and $\mathbf{Z}(\mathbf{s})$ function provides a possibility to quantify the result of operations on infinite numbers in terms of the achieved order of infinity and the analytic value.

\section{Conclusions}

The objective of this research is the analysis of infinity and the possibility of analytical interpretation of infinite numbers. The proposed approach attempts to develop a plural nature of infinity. The infinity is understood not as a single undefined large value of an absolute nature, but rather as a dynamic collection of multiple undefined values, which belong to a separate domain with its own mathematics applicable only to the infinite numbers. The actual infinite values may remain undefined, but the attributes of the undefined values can be elaborated. This research proposes to use order, class, and analytic values as infinity attributes, which can uniquely characterize the infinite numbers. The classification is based on the intensity of diverging functions, which approach infinity. For lower order of infinity, it is proposed to use the association with Riemann's zeta function for establishing analytic value of infinity.

The proposed classification of infinite domain allows definition of arithmetic operations on infinite numbers. The result of operations on infinite numbers can be presented as another infinite number with unknown value, but with known characteristics. These resulting characteristics are determined by the attributes of operands, and the analytic value can identify the result of arithmetical operation in infinite domain.

\section{References}

[1] Keisler, H. J. (1986). Elementary Calculus: An Approach Using Infinitesimals (2nd ed.).

[1] Keisler, H. J. (1994). The hyperreal line. Real Numbers, Generalizations of Reals, and Theories of Continua. 207-237. Kluwer Academic Publishers.

[2] Conway, J. H. J. H., \& Guy, R. K. (1996). The Book of Numbers, 283-284. New York: Springer-Verlag.

[3] Donald, E. K. (1974). Surreal Numbers. Reading. MA: Addison-Wesley Pub.

[4] Ehrlich, P. (2012). The absolute arithmetic continuum and the unification of all numbers great and small. The Bulletin of Symbolic Logic, 18(1), 1-45.

[5] Radjabli, K. (2016). Analysis of sparsely populated sets. Proceedings of ICPAM Conference. London.

[6] Abramowitz, M., \& Stegun, I. A. (1972). Riemann zeta function and other sums of reciprocal powers. §23.2. Handbook of Mathematical Functions with Formulas, Graphs, and Mathematical Tables, 807-808. 
[7] MacDonnell, J. F. (1989). Some critical points of the hyperpower function. International Journal of Mathematical Education, 20(\#2), 297.

[8] Goodstein, R. L. (1947). Transfinite ordinals in recursive number theory. The Journal of Symbolic Logic, $12,123-129$.

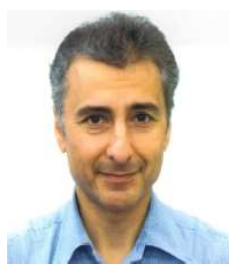

Kiamran Radjabli was born in 1959 in Baku, Azerbaijan. He earned his master of science and doctorate degrees in electrical engineering from Moscow Power Engineering Institute. He is a professional engineer licensed in Ontario, Canada. He provided consulting services to a number of electrical utilities in North America and abroad. He is carrying out research in the area of sparsely populated sets, conditions close to emptiness, and infinite space domains. Kiamran has over 20 years of experience in control systems, software development, and real time applications. 